\title{
Ictericia en recién nacidos de madres con Colestasia Intrahepática del embarazo. Influencia de la Leche Materna.1
}

\author{
1)r. Sergio Vaisman $W^{2}$ y Dr. Lujs Villavicencio F."
}

\begin{abstract}
Serum bilirubin levels in new borns from mothers with intrahepatic cholestasis of pregnancy
30 bewbons from un uthers with intrubepabc sholestisis af prestouncy ind 30 controls were studied. Senum bilimbiu levels were similar in bxth groups in the first six deys of life. Many simples of hunian milk inhibited bilinbiu glueuronyi mansferase activity in vitro, but no relation was found between inh ibitury capacity and sen num Jilinubur level of the newborns. A positive correlation was fomal hetween inhibition of glucuronyl transterase activity by humaul milk and total trete fatty acid content of tunlk samples.
\end{abstract}

La Colestasia Intrahepática del Embarazo (C.I.E.) es una enfermedad relativimente rara en la mayor parte de los paises, pero tiene una alta incidencia en Chile, ocuriendo en 3 a $4 \%$ de todos los embarazos. ${ }^{1}$

Esta condición en la madre se asocia con complicaciones en el feto y en el recién nacido, anmentando significativamente la mortalidad fetal y la morbilidad en el recién nacido. Estos factores hacen que la C.I.E., sea de gran interés para los Obstetras y Neonatólogos.

Los recién nacidos de madres con C.I.E. presentan una alta incidencia de Hiposia Neonatal, Hipoglicentia e Hiperbilimubinema. ${ }^{1}$ La hiperbilimbinemia se manifiesta por un aumento de la bilirnubina no conjugada en la primera semana de vida, con el consiguiente riesgo de Kemicterus. El o los mecanismos responsables de la hiperbilimulbinemia en estos recién nacidos es desconocido.

Espinoza describió la presencia, en orina de matdres con C.I.E., de un metabolito anormal, el pregnano-3 (alfa), 20 (Beta)-diol, ${ }^{2}$ El mismo metabolito fue encontrado por Arias y Gartner ${ }^{3}$ estudiando leches de madres que tenian recién nacidos con Ictericia por Leche Materna y demostraron que actuaba como inhibidor in vitro de la ylncuronil transferasa, enzima responsable de la conjugación de la bilimubina. ${ }^{3}$

Los objetivos de nuestro trabajo son estudiar en forma seriada, en la primera semana de vida, los

\footnotetext{
${ }^{1}$ Tratajo presentido en el XVI Congreso Intemacional de Pediatría, Barcelona, España; septiembre 1980. Trabajo financiado por Grant N. ${ }^{\circ}$ 6-121 The National Foundation March of Dimes. ${ }^{2}$ Medico Pediatra Maternidad Hospital Banos Luco, Facultad de Medicina Universidad ale Chile.

${ }^{3}$ Médico Obstetra Maternidad Hos pital Barros Luco.
}

niveles de bilimubinemia en un gnupo de recién nacidos de madres con C.I.E. y la capacidad inhibitoria que ejerce sobre la conjugación de bilimubina la leche de madres con esta enfermedad, para establecer el rol que puede jugar este factor en la etiologíi de la hiperbilirrubinentia neonatal en recién nacidos de madres con C.I.E.

\section{MATERIAL Y METODO}

Se tomó un grupo de 30 madres con C.I.E., diagnosticadas por anamnesis, exarnen fisico y pruebas de laboratorio, y sus recién nacidos alimentados con leche materna (Tabla 1). El grupo control lo constituyeron 30 madres con embarazos normales y sus recién nacidos alimentados al pecho. Todos los partos se atendieron en la Maternidad del Hospital Barros Luco (Tabla 2).

En ambos grupos se efectuaron bilimbinemias seriadas en los recién nacidos desde el segundo al sexto día de vida, utilizando una adaptación a micrométodo de la técnica de Malloy y Evelyn, ${ }^{4}$ En los recién nacidos se efectraron eshudios hematológicos incluyendo clasificación de grupo y $\mathrm{Rh}$,

\section{Tabla I}

Antecedentes de Madres con Colestasia

$$
(\mathrm{n}=30)
$$

Prerito: $30(100 \%)$

Coluria: 30 ( $10(0 \%)$

letericia: $20(66.7 \%)$

Primíparas: 21

Multíparas: 9
Comierizo: 33.03 semanas (promedio)

Comienzo: 35.6 semanas (promedio)

Colestasia en embarazo previo: 8 
Recién racidos de Marlres con Colestasia y Controles

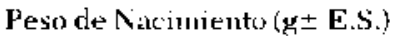

Edad Gestacional (semamas E E.S.)

$$
\text { Masculino }
$$

Sexo

$$
\text { Femenino }
$$

Apgar al Minuto (promedio)

Apgar a los 5 Minutus ipromedio!

prueba de Coontss, hematocrito, hemoglobina, recuento de reticulocitos y frotis sanguíneo.

En las madres se efectuó clasificación de grupo, Rh y estudio de función hepática incluyendo bilirrubinemiat, transaminasas y fosktasa alcalina. Se recogieron muestra de leche materna en el tercer, cuarto $y$ quinto día después del parto, y se efectuós en ellas estudio in vitro de inhibición de la conjugación de bilimubina, usando bilimbina cómo substrato, con una modificación de la técnica de Heirwegh y Meuwissen publicada por Gartner y Arias. ${ }^{5}$ Además se efectuó determinación colorimétrica del contenido de ácidos grasos libres de la leche materna en cada una de las muestras, expresando el resultado conno normal, positivo + , positivo ++ , positivo +++ , según el color de la reacción, que estai dado por la cantidad total de ácidos grasos libres (Técnica de Croiffon y Couchoud) ${ }^{6}$

En ambos grupos se eliminaron aquellos recién nacidos con causas conocidas que exageran la hiperbilirubinemia neonatal, incluyendo hemólisis, infeccion, cefalohematomas, grandes equimosis, hipoxia e hipoglicemia.

\section{RESULTADOS}

Niveles de Bilirrubinemia en el Recièn Nacido: En los primeros seis dias de vida, los recién nacjdos de madre con C.I.E. no presentan diferencias significativas en sus niveles de bilirrubina sérica en relación al grupo control (Figura 1 ).

Inhibición de la Actividad de Glucuronil Transferasa: Tanto en los recién nacidos de madres con C.I.E. como en el grupo control, se encuentra un número importante de leches inhibitorias. Sin embargo, la capacidad de inhibición de esas leches no se correlaciona con los niveles de bilirrubinemia de los recién nacidos (Figura 2).

Contenido de Acidos Grasos Libres de la Leche Materna: Se encuentra una correlación directa en-

$$
\begin{gathered}
\text { Control } \\
(1)=30\} \\
3256 \pm 7.3 \\
39.2 \pm .25
\end{gathered}
$$

20

$$
\begin{array}{r}
10 \\
7.93 \\
8.86
\end{array}
$$

\section{Figura 1}

Bilimulinemia en Reclén Nacidos de Madres con Colevtasia Intrahepatica del Embarazo y Grupo Contol en primeros seis días de vida (promedio \pm Error Standard).

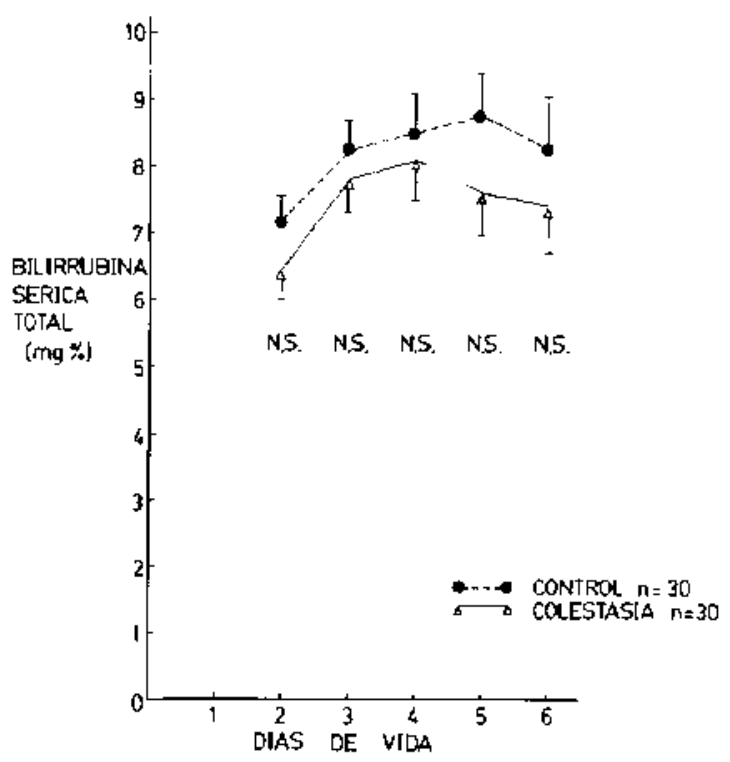

tre el coutenido total de ácidos grasos libres de las leches estudiadas y su capacidad inhibitoria de la conjugación de bilirrubina, independientemente de si se trata de madres con C.I.E. o madres del grupo control (Figura 3).

\section{DISCUSION}

Los resultados obtenidos en este estudio $11 \%$ concuerdan con lo publicado anteriomente en el sentido de que los recién nacidos hijos de madres con C.I.E. presentan hiperbilimubinemias mayores que el grupo control en la primera semana de vida. Aún más, en nuestro trabajo el grupo en eshidio cursa con niveles leventente inferiores a 
Figura 2

Inhibición de la actividad de la enzima glucuronil transferasa por Leche Matena y Bilirrubinemia Máxirna en Reciér Nacidus de Madres con Colestasia y Gimpo Control.

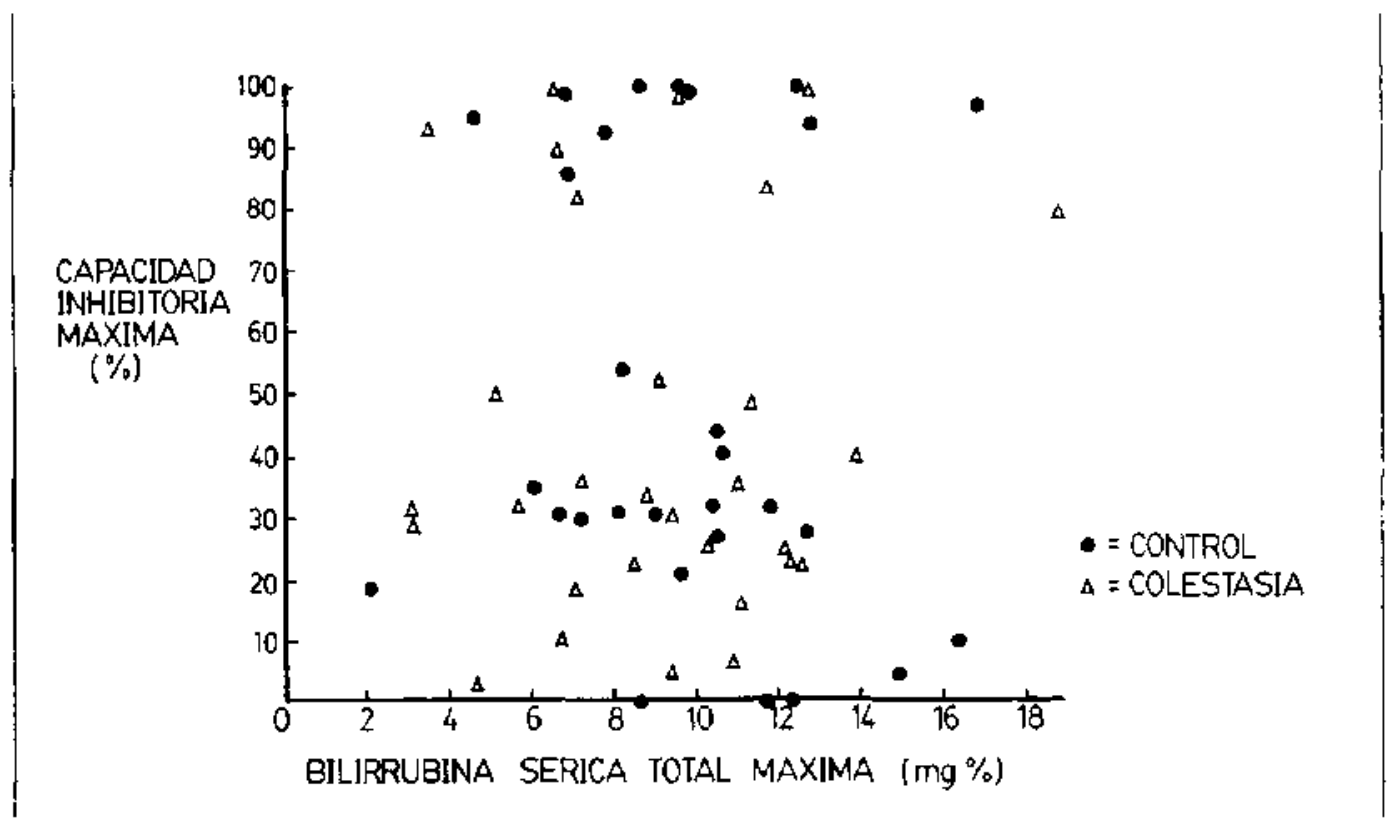

Figura 3

Inhibición de la actividad de la enzina glucuromil transferasa por Leche Materna y contenido de Acjolos Grasus Libres de las Muestras de Leche.

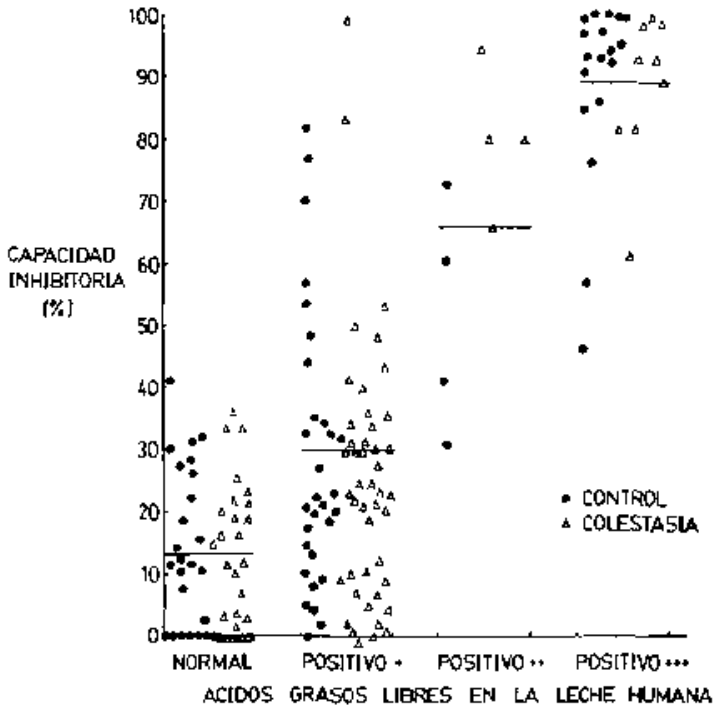

Son recién nazcidos de témino pero con una gesta. ción significativamente menor que el grupo controbl. A pesar de ello, la evolución de su bilirrubinemia no difiere en forna significativa.

No encontramos una relación entre leches inhibitorias y niveles de bilirrulsinemia en los primeros seis días de vida, pero sí encontramos una correlación entre la concentracion total de acidos grasos libres y la capacidad inhibitoria de esas leches sobre la conjugacion de bilitubina in vitro. Se sabe que algunos ácidos grasos libres como el linoleico, linolénico y araquidónico son inhibidores potentes dela actividad de la enzitua glucuronil transferasa, ${ }^{7}$ la enzima que conjuga la bilimobina.

El próximo paso obligatorio para continuar este eshudio es deteminar la concentración de cada ácido graso libre en la leche materna para ver si existe alguna correlación entre una concentración elevada de un ávido graso libre especifico, lia capacidad inhibitoria de la leche $y$ la ictericia neonatal.

\section{RESUMEN}

Se estudian 30 recién nacidos de madres con los controles pero ésta diferencia no es significativa. El grupo con colestasia tuvo una interrupción de la gestación, en promedio, a las 38.3 semanas. 
colestasia intrahepática del embarazo y 30 controles. Ambos grupos tienen niveles comparables de bilirrubinemia en los primeros seis días de vida. Se encontró un número inportunte de muestras de leche materna y intiben la conjugación de bilimubina in vitro, pero no hubo relación con los niveles de bilirrubina sérica de los recién nacidos. La capacidad inhibitoria de las leches matenas es directamente proporcional al contenido total de ácidos grasos libres de las muestras analizaclas.

\section{REFEHENCLAS}

I Erazo, R.: Colestasia lntrahepática de] Embarazo. Neonatologia 111: 9: 1975.
Espinoza, J., Barnaft, L. and Schnadt. E.: Phenolsarbital in Cholestasis of Preghaney. An. J. Obstet. Gynec. 119: 234: 1974.

"Arias, I.M., Gartner, L.M., Seifter, S. and Furman, M.: Prolonged Neonatal Untoujugated Huperbilimubinemia associated with Breast Feeding and a steroid. Pregrame-3 (alpha), 20 (Beta) djol in Maternal S(ilk that inhibits glucuronide fornuation in vitro. J. Clin. Invest. 43: 2037; 1964 .

${ }^{4}$ Malloy, H.I. and Evelyn, K.A.: The determisation of Bilintbin with the Photoelectric Colorimeter. J. Biul. Chen. 119:481 1937 .

¿Gartner, L.M. and Arias I.M.: Homnonal control of hepatic bilinubin transport and eonjugation. An. J. Physiol. 202: 1091; 1972.

6 Goriffon, R., Couchuted, M.: Procédés de milcrodosage des acides yras non saturés par le bleude Nil. Ann. Biol. Cliru. 11:327. 1953.

TVaisman, S.. Ictericia por alimcutacion con Leche Muterna. Veonatología 6: 22: 1975. 